\title{
Sistema tutor afectivo para el aprendizaje de las matemáticas usando técnicas de gamificación
}

\author{
Ramón Zatarain-Cabada, María Lucia Barrón-Estrada, \\ Jorge García-Lizárraga \\ Instituto Tecnológico de Culiacán, Sinaloa, \\ México \\ \{rzatarain, lbarron, 86170331\}@itculiacan.edu.mx
}

\begin{abstract}
Resumen. De acuerdo a evaluaciones internacionales en los niveles básicos de educación, México se encuentra entre los países que presentan menor puntuación en lo que respecta al aprendizaje de las Matemáticas. Este artículo presenta el diseño de un Sistema Tutor Afectivo para el Aprendizaje de las Matemáticas (STAAM), el cual relaciona situaciones de aprendizaje con ejercicios matemáticos orientados al plan de estudios oficial vigente. El tutor incluye un reconocimiento bimodal de emociones, una técnica de gamificación para la motivación del estudiante y una retroalimentación en cada ejercicio de acuerdo al estilo de aprendizaje del usuario. La puntuación de cada ejercicio se determina con base a variables como el tiempo de respuesta, el número de intentos y el nivel del ejercicio. Se incorporó el estado emocional del estudiante para determinar el siguiente ejercicio. Los resultados obtenidos con estudiantes, mostraron un grado satisfactorio de aceptabilidad como también un avance significativo en ciertos aprendizajes esperados del plan de estudios.
\end{abstract}

Palabras clave: Computación afectiva, sistemas tutores inteligentes, gamificación, software educativo.

\section{Affective Tutoring System for Learning of Mathematics Using Gamification Techniques}

\begin{abstract}
According to international assessments in basic education levels, Mexico is among the countries with the lowest score in regard to the learning of mathematics. This paper presents the design of an Affective Tutoring System for Learning Mathematics (ATSLM), which relates learning situations with mathematical exercises oriented to the current official curriculum in Mexico. The tutor includes a bimodal emotion recognition, a gamification technique for student motivation and feedback for each exercise according to the user's learning style. The score for each exercise is determined based on variables such as response time, number of attempts and the exercise complexity level. Student's emotional state is incorporated to determine the next exercise. The results obtained with students, showed a satisfactory degree of acceptability as a significant advance in certain learning outcomes of the curriculum.
\end{abstract}

Keywords: Affective computing, intelligent tutoring systems, gamification, educational software. 


\section{Introducción}

De acuerdo con los resultados de PISA 2012, el panorama en matemáticas de los jóvenes mexicanos es insatisfactorio ya que no alcanzan el nivel de competencias básico (nivel 2) en matemáticas (promedio OCDE: 23\%). El alumno promedio en México obtiene 413 puntos en matemáticas. El puntaje promedio en la Organización para la Cooperación y el Desarrollo Económico (OCDE) es de 494, una diferencia que equivale a casi dos años de escolaridad [1].

Los Sistemas Tutores Afectivos (STA) son Sistemas Inteligentes que incorporan la habilidad para reconocer el estado afectivo de los estudiantes permitiendo al usuario interactuar con ejercicios que estimulen su estado emotivo [2].

Las técnicas de gamificación (gamification) se usan en la educación con el propósito de disminuir los abandonos, la falta de motivación y la falta de compromiso en las actividades de aprendizaje [3].

El uso de un STA que integre el reconocimiento emocional, técnicas de gamificación y estilos de aprendizaje [4], es una alternativa para el mejoramiento del aprendizaje de las matemáticas [5].

Este trabajo presenta un Sistema Tutor Afectivo para el Aprendizaje de las Matemáticas (STAAM) de primer grado de secundaria, usando ejercicios matemáticos de acuerdo al plan de estudios vigentes, con la incorporación de técnicas de gamificación que permiten motivar al estudiante, con técnicas de reconocimiento de emociones bimodal (texto y rostro), así como el uso del estilo de aprendizaje del usuario que permite dar una retroalimentación en cada ejercicio para favorecer su proceso de aprendizaje.

La implementación de STAAM para alumnos de Primer Grado de Secundaria permitirá:

- Despertar el interés del estudio de las matemáticas mediante el uso de un tutor afectivo implementando técnicas de gamificación.

- Fortalecer el aprendizaje de temas de difícil comprensión usando estrategias didácticas con retroalimentación orientadas a su estilo de aprendizaje.

- Integrar competencias matemáticas en alumnos de primer grado en su ingreso al nivel de secundaria.

- Evaluar el desenvolvimiento de los alumnos en cada uno de los aprendizajes esperados que contempla los 5 bloques del plan de estudios vigentes, identificando fortalezas y debilidades en cada uno de ellos.

De esta manera, la implementación de STAAM desarrolla una nueva forma de aprendizaje de las matemáticas de manera más motivante e innovadora.

Actualmente existe gran diversidad de herramientas de apoyo para el aprendizaje de las matemáticas que no contemplan la emoción de los usuarios al momento de utilizarlas. Diversos estudios demuestran que el estado emocional del usuario está estrechamente relacionado con procesos cognitivos como el aprendizaje [6], lo que puede favorecer o bloquear este proceso.

STAAM integra una plataforma para el reconocimiento de emociones en expresiones faciales y textuales denominado PREMOC. 
Este artículo está dividido en 6 secciones. En la segunda sección se describen trabajos relacionados. En la tercera sección se presenta la arquitectura de STAAM así como el trabajo realizado en el desarrollo del reconocedor PREMOC que se utilizó en la investigación. En la cuarta sección se describe el funcionamiento del tutor. En la quinta sección se muestran resultados del software propuesto. Por último, en la sexta sección se describen las conclusiones del trabajo.

\section{Trabajos relacionados}

Existen diversos trabajos sobre el aprendizaje de las matemáticas que se enfocan solamente en la resolución de problemas matemáticos como MathTutor [7]. Esta herramienta es un Sistema Tutor Inteligente (STI) basado en la Web diseñado para ayudar a los estudiantes de 6to grado de primaria, 1ro y 2do de secundaria, para aprender Matemáticas realizando ejercicios ${ }^{1}$.

C. González y A. Mora utilizan elementos de gamificación como componentes principales del sistema EMATIC (Mathematics Education through ICT) [8], el cual es un STI orientado a tablets y dispositivos móviles para la enseñanza de operaciones matemáticas básicas.

Por otra parte, E. Melis presenta un STI (ActiveMath) que proporciona recursos interactivos para mejorar y enriquecer la enseñanza y el aprendizaje de las matemáticas [9]. El STI es un sistema tutorial inteligente basado en la Web para las matemáticas que contiene actividades que pueden ser utilizadas tanto por los alumnos individuales, grupos pequeños o con toda la clase utilizando un proyector o una pizarra interactiva.

MathSpring (antes Wayang Outpost) [10], es un Sistema Tutor Inteligente diseñado para aprender junto con el estudiante. Usando multimedia interactiva, MathSpring ayuda a preparar a los estudiantes de secundaria y preparatoria para los exámenes estandarizados de matemáticas como el SAT, MCAS y CA-Star.

Animal Watch [11], es un STI que fue desarrollado con dos objetivos en mente: a) enseñar con eficacia aritmética y pre-álgebra para estudiantes de primaria y secundaria, b) aumentar la confianza, el valor y el gusto de las matemáticas de los jóvenes estudiantes.

S. D’Mello, A. Olney, C. Williams, y P. Hays desarrollaron un tutor que utiliza un rastreador ocular comercial para monitorear los patrones de la mirada de un estudiante e identificar cuando está aburrido, llamado Gaze Tutor [12]. Este STI tiene como objetivo promover la participación y el aprendizaje mediante la detección de forma dinámica y la respuesta al aburrimiento.

La principal aportación de STAAM es la integración en el aprendizaje de las Matemáticas de las técnicas de reconocimiento bimodal de emociones, con técnicas de gamificación y estilos de aprendizaje. Otra aportación importante de nuestra herramienta, es que fue creada tomando en cuenta el programa oficial de estudios de Matemáticas vigente en México, por lo que se adapta completamente a los ejercicios y problemas aplicados y usados por los profesores y estudiantes del nivel básico.

\footnotetext{
${ }^{1}$ https://mathtutor.web.cmu.edu/
} 


\section{Desarrollo del reconocedor bimodal de emociones}

Las emociones se han considerado como un factor importante en la toma de decisiones [14], y el aprendizaje de las matemáticas no es la excepción. Por esta razón se consideró importante la incorporación del estado emocional del estudiante al momento de llevar a cabo los ejercicios matemáticos, lo cual permite dirigir el aprendizaje de acuerdo a su estado afectivo.

\subsection{Arquitectura del STAAM}

La estructura del Sistema Tutor Afectivo (Figura 1) presenta el funcionamiento general de STAAM desde la interfaz de usuario, los controladores (Afectivo, Gamificador y de Ejercicio), teniendo como aportación importante la incorporación del estado emocional para determinar el siguiente ejercicio a realizar por el estudiante, así como el controlador gamificador.

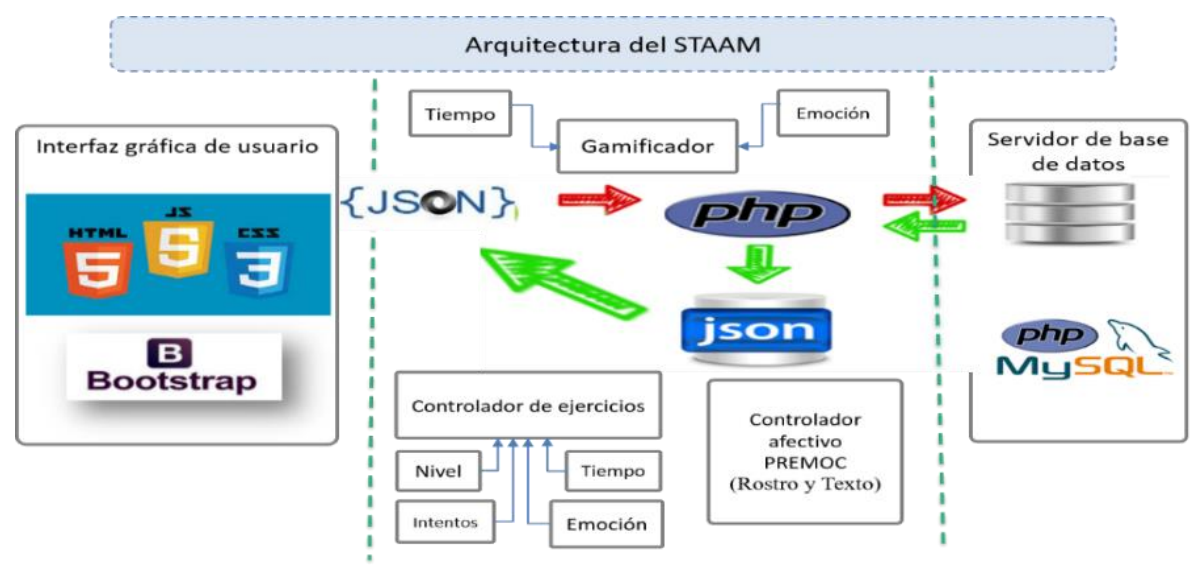

Fig. 1. Estructura del Tutor.

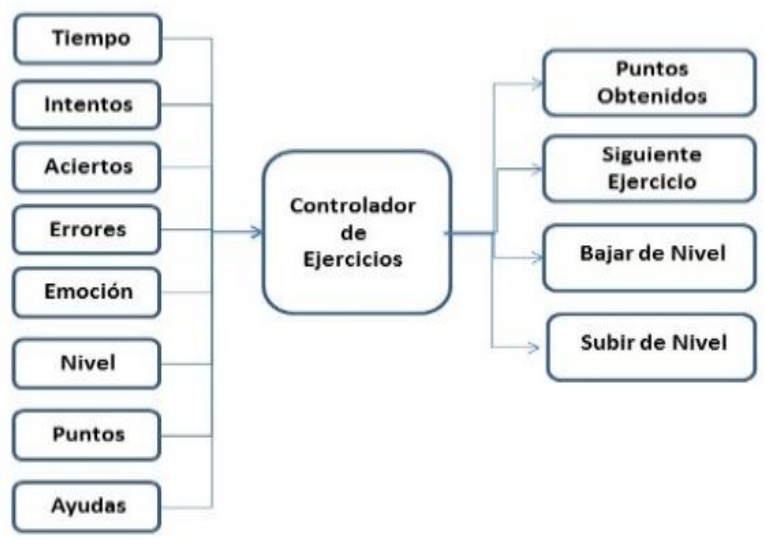

Fig. 2. Controlador de ejercicios. 
STAAM integra la persistencia de la información en una base de datos en un servidor apache usando intercambio de información mediante objetos JSon.

\subsection{Controlador de ejercicios}

El controlador de ejercicios considera 8 variables importantes (Figura 2).

El controlador de ejercicios implementa 4 algoritmos (Figura 3), que nos permiten determinar: los puntos obtenidos por cada ejercicio, el siguiente ejercicio a realizar y el cambio de nivel de aprendizaje (bajar o subir de nivel).
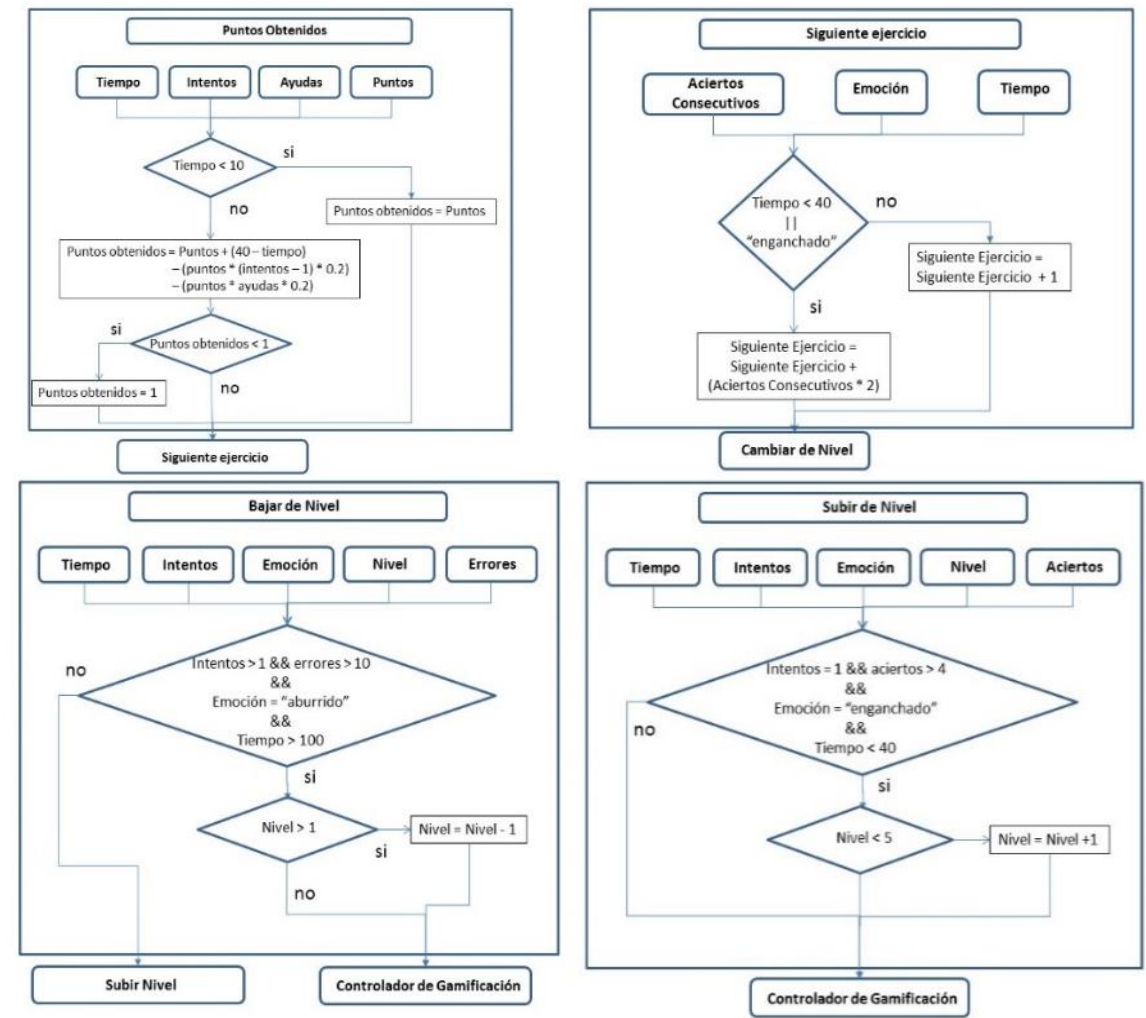

Fig.3. Algoritmos implementados en el controlador de ejercicios.

\subsection{Controlador de gamificación}

El controlador gamificador (Figura 4) se encarga de calcular los puntos extras o bonos que se identifican con estrellas personalizadas con una imagen, acorde a la emoción reconocida. Estos puntos extras o bonos se obtienen considerando el tiempo de respuesta (entre menos tiempo mayor puntaje), el número de aciertos realizados de forma consecutiva (entre mayores aciertos consecutivos se obtendrá mayor puntaje) y la emoción obtenida al momento de responder (cuando la emoción es “enganchado"). 
Cuando la emoción detectada es "frustrado", el tutor ofrece ayuda de retroalimentación del ejercicio propuesto, de acuerdo al estilo de aprendizaje del usuario y ejecuta ejercicios de menor nivel cognitivo si las últimas cuatro respuestas son erróneas y el tiempo de respuesta es mayor a 40 segundos.

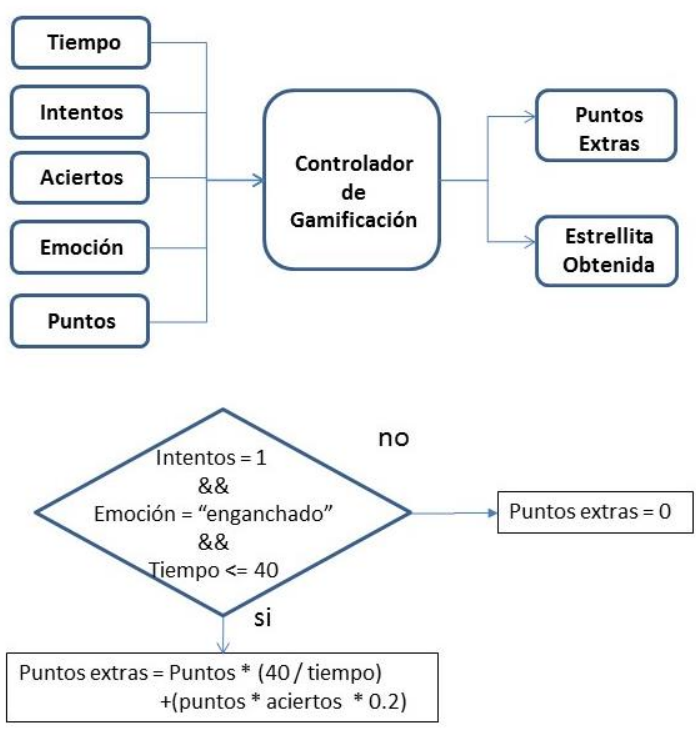

Fig. 4. Implementación del controlador de gamificación.

\subsection{PREMOC}

PREMOC es una plataforma en línea que ofrece reconocimiento de emociones a través de un servicio Web. La comunicación entre PREMOC y STAAM es a través de formato JSON, en donde el tutor envía sus datos de entrada por medio de un POST en este formato y recibe la emoción en modo "studen". En modo student, el PREMOC dará como respuesta el valor de una emoción tomando en cuenta el ámbito de aprendizaje (contexto educativo). Estas emociones son enganchado, aburrido, frustrado y neutral.

PREMOC tiene definidos cada uno de los reconocedores de forma autónoma, los cuáles reciben datos como: la imagen del rostro, voz, texto y/o información cerebral, este lo procesa, y obtienen la emoción. Los reconocedores fueron creados por separado en el laboratorio de la Maestría en Ciencias de la Computación del Instituto Tecnológico de Culiacán.

\subsection{Reconocimiento de emociones en texto}

Para el reconocimiento de emociones en texto, PREMOC utiliza un algoritmo semántico denominado ASEM. Este algoritmo está basado en palabras claves llamadas "keywords", pues contiene una colección de palabras previamente etiquetadas a una emoción. 
Para llevar a cabo el algoritmo ASEM se cuenta con 5 diferentes corpus, con diferentes funciones cada uno de ellos: stop words, palabras impropias, corpus emocional, new words y corpus semántico.

- Stop words. Es una colección de palabras que no influyen en la emoción de la oración, como artículos, preposiciones, entre otros. Las palabras que están aquí son eliminadas de la oración a evaluar.

- Palabras impropias. Es una colección de, como su nombre lo dice, palabras impropias, que están calificadas a una emoción y su factor de probabilidad afectiva a dicha emoción (FPA) donde $0<$ FPA $<1$.

- Corpus emocional. Es una colección de palabras, que están calificadas a una emoción y su FPA a dicha emoción, donde $0<$ PFA $<1$.

- New words. Aquí se coleccionan las palabras nuevas que no se tienen en el corpus emocional. Esto es para el mantenimiento del algoritmo ya que las nuevas palabras encontradas aquí serán calificadas cada cierto tiempo por un experto. El experto determinará a que emoción pertenece esa palabra y su FPA.

- Corpus semántico. Es una colección de palabras que potencializa o inhibe el valor FPA semánticamente. Por ejemplo, la palabra muy potencializa el valor FPA de la siguiente palabra.

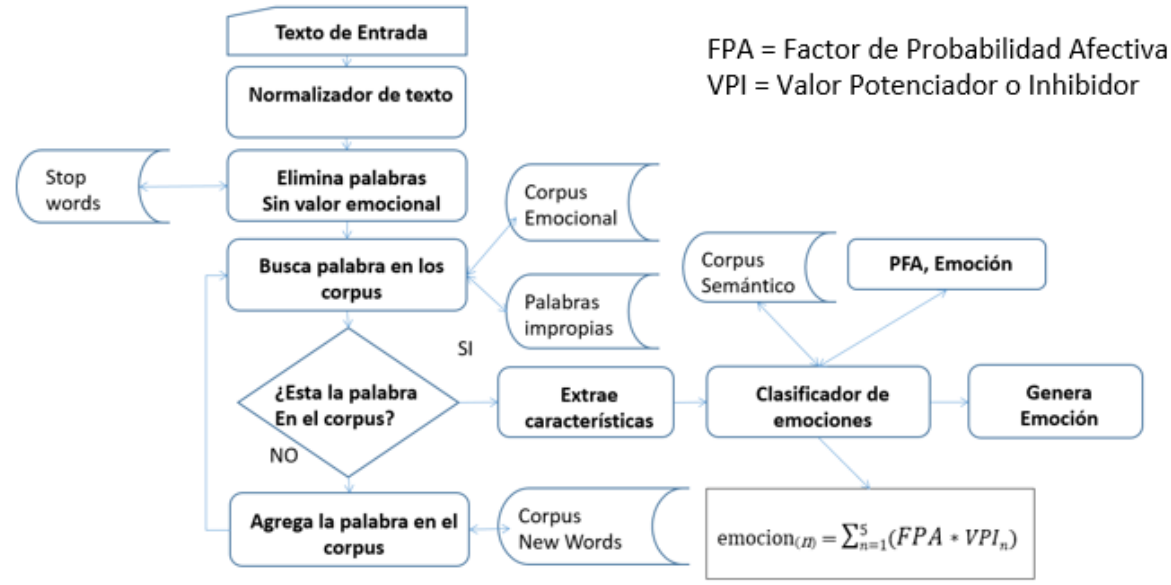

Fig. 5. Algoritmo ASEM.

Como se muestra en la imagen (Figura 5) el algoritmo ASEM funciona de la siguiente manera:

- Se recibe un texto de entrada.

- Se normaliza el texto quitando palabras que no agregan valor emocional usando el corpus Stop Words.

- Se busca cada palabra en el corpus emocional para obtener su emoción y su FPA. Si no está, se busca en el corpus palabras impropias. Si no está, se agrega al corpus new words para que sea más adelante clasificada por un experto. En la evaluación presente, dicha palabra es ignorada para la clasificación de la emoción en el texto. 
- Se buscan palabras en el corpus semántico que puedan potencializar o inhibir los valores FPA ya obtenidos.

- Se calcula el valor de cada una de las 4 emociones del modo student (enganchado, frustrado, sorprendido y neutral) con la fórmula:

$$
\operatorname{emocion}_{(n)}=\sum_{n=1}^{5}\left(F P A * V P I_{n}\right)
$$

Se selecciona la emoción con el mayor valor obtenido y esa emoción se regresa al usuario. La sintaxis en JSON para indicar que lo que se envía es un texto es la siguiente:

$$
\text { txt:"texto a evaluar". }
$$

\subsection{Reconocimiento de emociones en rostros}

El reconocedor de expresiones faciales en PREMOC consiste en clasificar las imágenes recibidas y regresar una de las 4 emociones en modo student.

La fase de ejecución en línea funciona de la siguiente manera como muestra en la Figura 6.

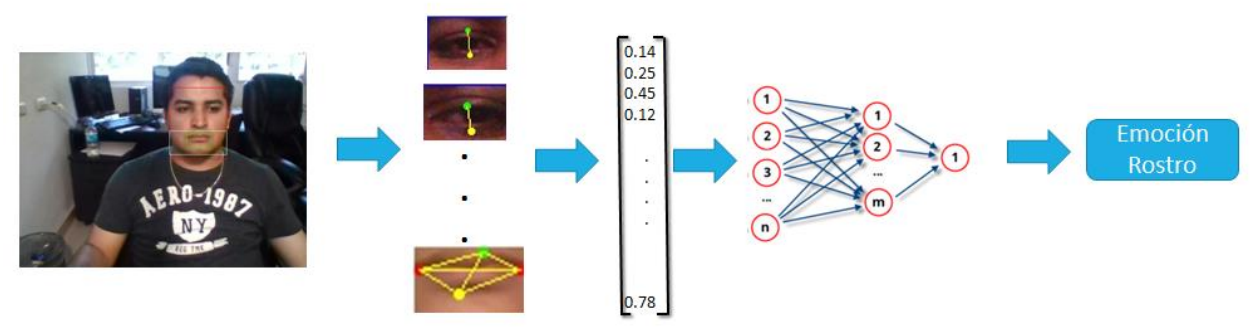

Fig. 6. Algoritmo del reconocedor de emociones en rostros.

- Recibe una imagen.

- Detecta el rostro en la imagen.

- Repite los siguientes pasos para boca, ojo izquierdo, ojo derecho, ceja izquierda, ceja derecha, lo llamaremos objetivo:

- Ubicar dentro del rostro posible zona de ubicación del objetivo, se llamará región de interés.

- Dentro de la región de interés aplicar un método de OpenCV para buscar en concreto el objetivo que estamos buscando.

- Ya con el objetivo encontrado, se le aplican filtros de OpenCV [30] como se muestra en la figura 3 para determinar los puntos clave en el rostro.

- Se crea un vector de características de 10 elementos, donde cada uno es una distancia euclidiana entre diferentes puntos clave ubicados en el rostro.

- Se ingresa el vector de características a la red neuronal ya entrenada para que lo clasifique a una de las emociones.

- Regresa la emoción obtenida.

La sintaxis en JSON para indicar que lo que se envía es una imagen es la siguiente: 


$$
\text { img:"Base64_imagen". }
$$

El reconocedor de emociones en rostros la convierte de nuevo en una imagen para procesarla en modo "student".

\section{Implementación de STAAM}

STAAM contiene diversos ejercicios divididos en 5 bloques, donde cada bloque puede contener hasta 12 secuencias didácticas. Cada secuencia didáctica puede tener hasta 12 temas principales y cada tema contiene un banco de reactivos de 1 a 999 ejercicios. (Figura 7).

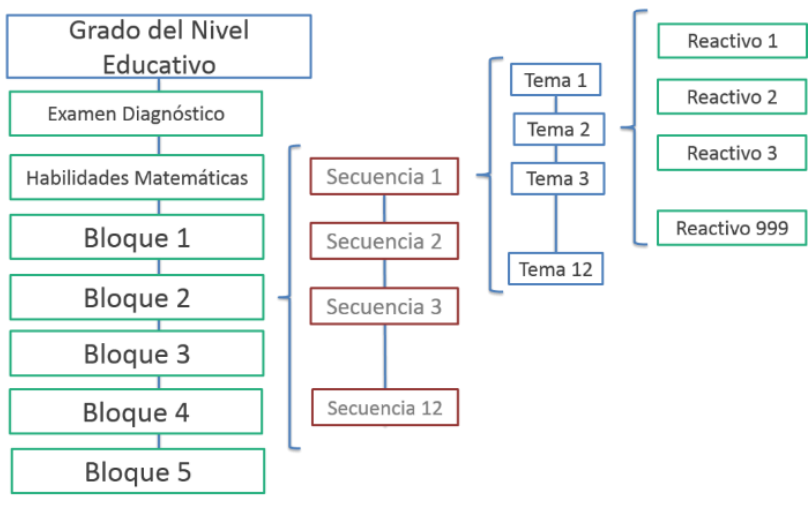

Fig. 7. Características del STAAM.

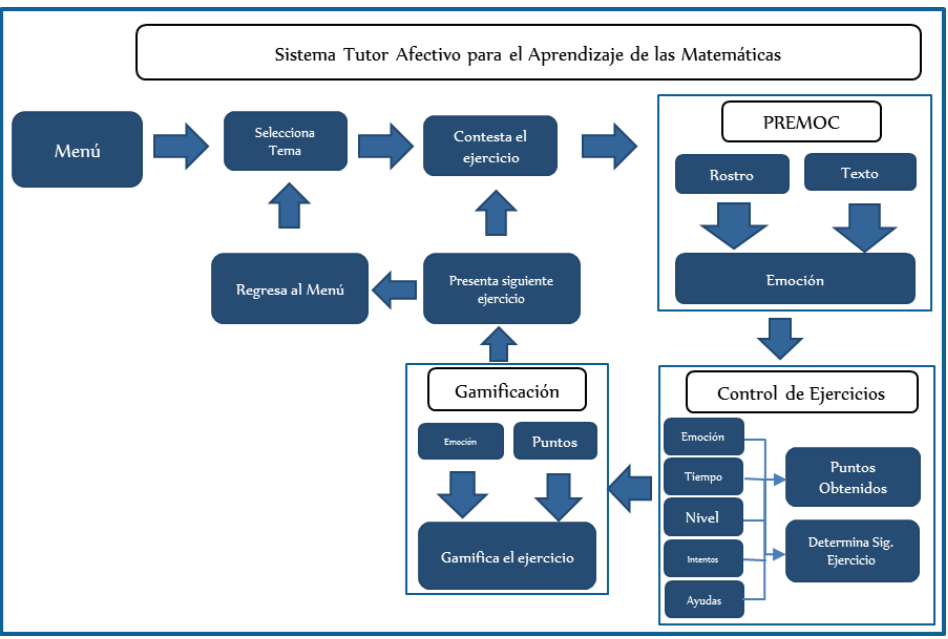

Fig. 8. Vista de controladores.

El estudiante resolverá los problemas que el STI le proporcione de acuerdo al determinado por el controlador de ejercicios. Cabe destacar que los contenidos que se abordan en cada uno de los ejercicios corresponden al plan de estudios vigentes. A 
continuación, se muestra la arquitectura de los controladores principales de STAAM (Figura 8).

STAAM presenta el menú principal donde el estudiante selecciona el tema y la herramienta, usando el banco de ejercicios, le presenta el problema a realizar. Al momento de responder se toma una foto del rostro del estudiante para identificar su estado emocional, mediante la plataforma PREMOC. Ésta regresa la emoción del estudiante, la cual puede ser: enganchado, frustrado, sorprendido o neutral.

El controlador de ejercicios determina los puntos obtenidos por el estudiante, el siguiente ejercicio y el cambio de nivel de aprendizaje.

El controlador de gamificación considera la emoción y los puntos obtenidos para determinar el tipo de estrella ganado por el usuario, así mismo muestra el avance del ejercicio, los puntos globales obtenidos y la calificación obtenida por cada tema de acuerdo a la siguiente fórmula. (Figura 9)

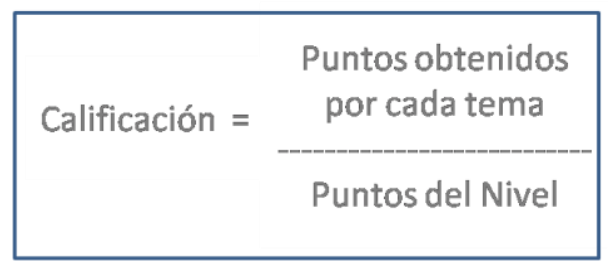

$$
\begin{aligned}
& \text { Nivel } 1=10 \text { pts } \\
& \text { Nivel } 2=20 \text { pts } \\
& \text { Nivel } 3=40 \text { pts } \\
& \text { Nivel } 4=70 \text { pts } \\
& \text { Nivel } 5=100 \text { pts }
\end{aligned}
$$

Fig. 9. Fórmula para la Calificación.

Por último, se muestra el siguiente ejercicio a evaluar y el proceso se repite.

\section{Pruebas y experimentos}

Actualmente se están haciendo experimentos con grupos de 1er año de secundaria (grupo A de experimentación y grupo B de control) obteniéndose resultados satisfactorios donde el uso de la herramienta ha despertado un gran interés por parte de los profesores y de los estudiantes.

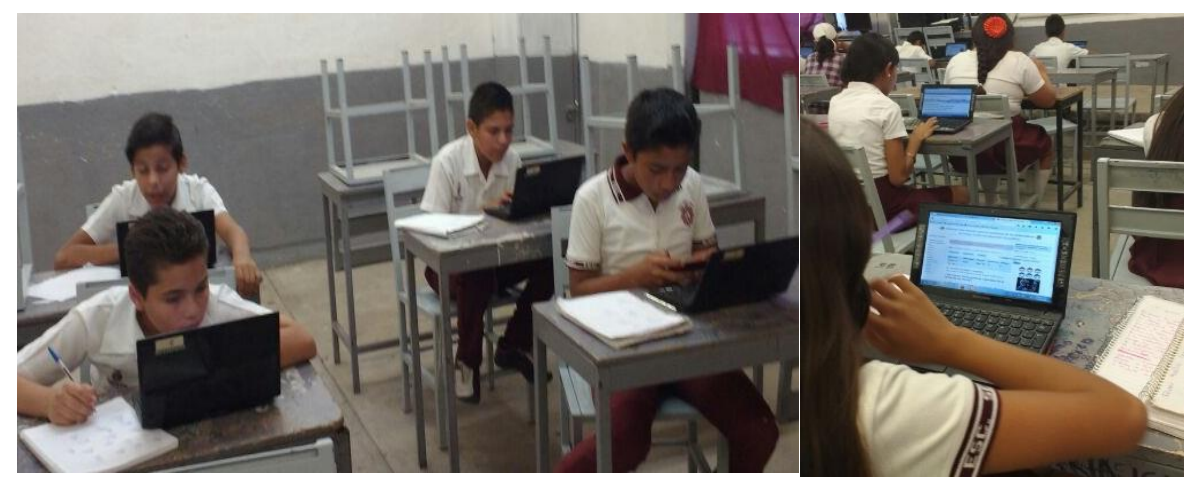

Fig. 10. Grupo de experimentación 1. 
En una prueba inicial (Fig. 10), participaron 19 estudiantes quienes terminaron los 20 ejercicios propuestos en un tiempo mínimo y con pocos errores, lo que permitió el aprendizaje de los ejercicios motivando a los estudiantes en la utilización del STAAM.

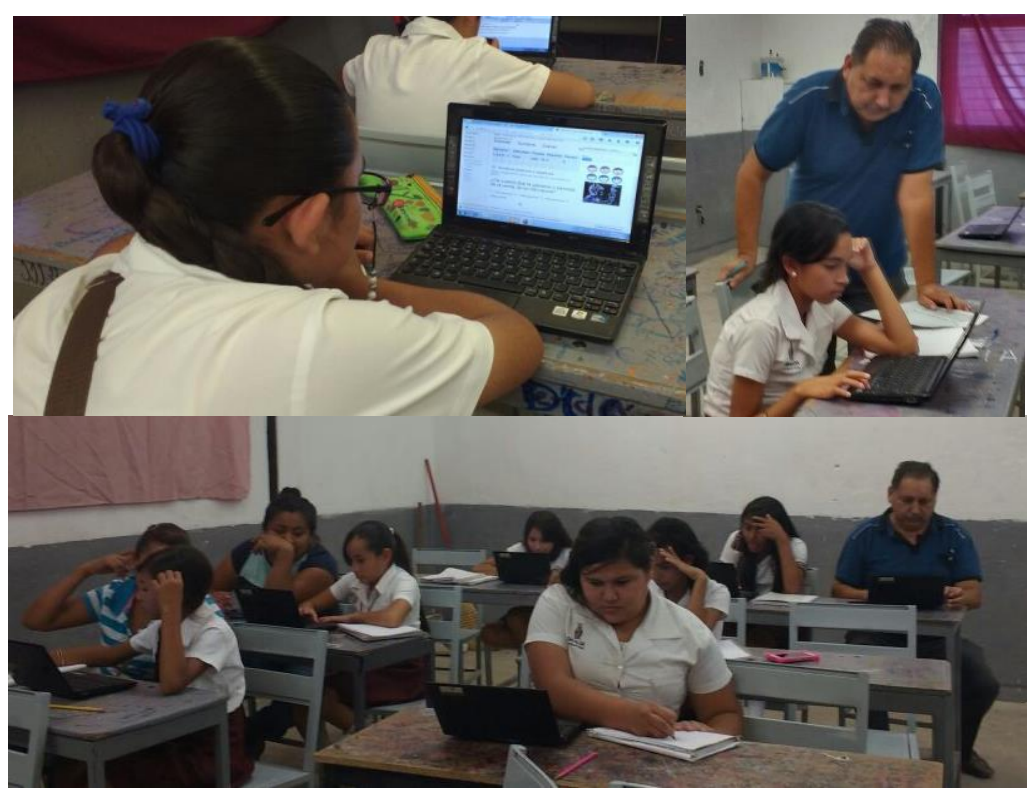

Fig. 11. Grupo de experimentación 2.

En una segunda intervención (Fig. 11), se invitó a madres de familia para que observarán la realización de ejercicios usando el tutor. Aquí participaron un grupo de 12 estudiantes, 8 mujeres y 4 hombres. Se trabajó con 40 ejercicios y se dispuso de un módulo de $45 \mathrm{~min}$.

En la Tabla 1 se muestran los resultados obtenidos por 9 estudiantes escogidos del \# lista (se escogieron los \# de lista par).

Tabla 1. Resultados obtenidos.

\begin{tabular}{cccccc}
\hline Estudiante & Genero & $\begin{array}{c}\text { Tiempo prom. } \\
\text { De respuesta }\end{array}$ & $\begin{array}{c}\text { \# de intentos } \\
\text { totales }\end{array}$ & $\begin{array}{c}\text { Emoción } \\
\text { Predominante }\end{array}$ & $\begin{array}{c}\text { Nivel del } \\
\text { Ejercicio }\end{array}$ \\
\hline E01 & M & $43 \mathrm{seg}$ & 23 & Neutral & Fácil \\
\hline E02 & M & $48 \mathrm{seg}$ & 25 & Frustrado & Fácil \\
\hline E03 & F & $47 \mathrm{seg}$ & 24 & Neutral & Fácil \\
\hline E04 & M & $33 \mathrm{seg}$ & 21 & Neutral & Fácil \\
\hline E05 & F & $38 \mathrm{seg}$ & 22 & Engancil \\
\hline E06 & M & $43 \mathrm{seg}$ & 22 & Frustrado & Fácil \\
\hline E07 & F & $41 \mathrm{seg}$ & 22 & Engácil \\
\hline E08 & F & $45 \mathrm{seg}$ & 24 & & Fácil \\
\hline E09 & M & $39 \mathrm{seg}$ & 22 & & Eado \\
\hline
\end{tabular}


El tiempo de respuesta promedio de los estudiantes se eleva considerablemente cuando no se les permite el uso de calculadora llegando a ser por arriba de los 120 segundos en más del $80 \%$ de la población ocasionado por la dificultad de poder realizar las operaciones básicas de forma mental, mejorándose significativamente esta variable cuando si se utiliza la calculadora.

Para acceder al tutor es necesario autentificarse, si el usuario es nuevo, se procede a registrarse, de lo contrario debe ingresar su usuario y contraseña.

$\mathrm{Al}$ entrar el estudiante, debe seleccionar el bloque con el que se trabajará, así como el tema y subtema.

Una vez escogido el subtema, se procede a realizar los ejercicios correspondientes mostrándose en su caso, diferentes estrellas que identifican los ejercicios resueltos correctamente, los puntos y la emoción obtenida en el momento de la respuesta. (Figura 12).

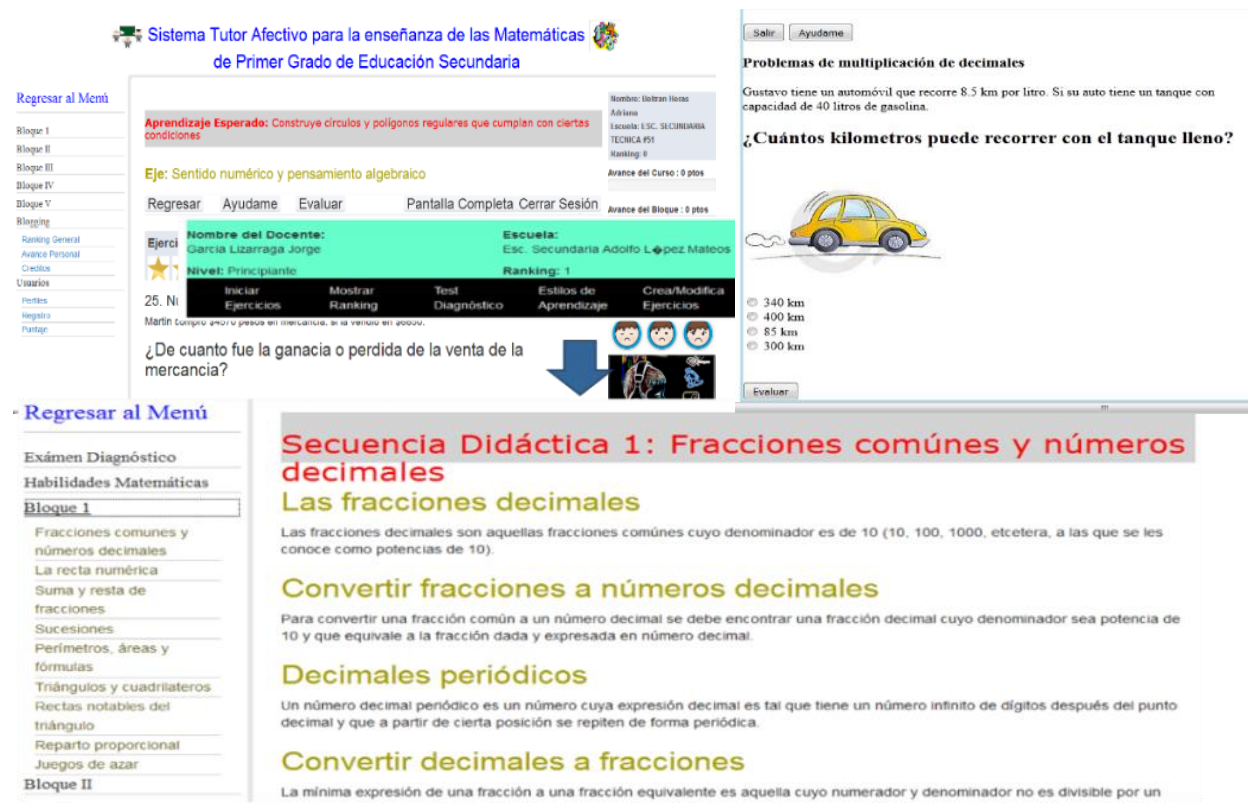

Fig. 12. Pantalla de ejercicios.

Tabla 2. Estrellas indicadoras de los puntos ganados de acuerdo al tiempo y estado emocional.

\begin{tabular}{|c|c|c|c|c|c|c|c|}
\hline $\begin{array}{l}\text { Estrella } \\
\text { ganada }\end{array}$ & & 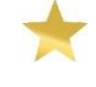 & $\stackrel{1}{=}$ & & ( & & \\
\hline Emoción & frustrado & neutral & neutral & enganchado & enganchado & enganchado & sorprendido \\
\hline Tiempo & Mucho & Mucho & $\begin{array}{l}\text { Poco, } \\
\text { Regular }\end{array}$ & Mucho & Regular & Poco & $\begin{array}{l}\text { Poco, } \\
\text { Regular }\end{array}$ \\
\hline $\begin{array}{l}\text { Puntos } \\
\text { Extras }\end{array}$ & 0 & 0 & 0 & \multicolumn{4}{|c|}{$\begin{array}{c}\text { Ptos extras }=\text { ptos ejerc }+ \text { tiempo ejerc. }- \text { tiempo } \\
\text { respuesta }\end{array}$} \\
\hline
\end{tabular}

*Nota: Los puntos se obtienen solamente cuando el ejercicio se respondió correctamente. 
Cada ejercicio realizado se identifica con icono representativo mediante una estrella la cual ésta relacionada a un estado emocional y el tiempo que tardó en contestar correctamente dicho ejercicio (Tabla 2).

\section{Conclusiones}

Se puede concluir que la implementación del tutor vendrá a favorecer el aprendizaje de las matemáticas ya que desafía al estudiante en cada uno de los temas propuestos manteniéndolos motivados en cada momento lo que permite su integración al tutor y desarrollar sus habilidades matemáticas.

El STAAM se encuentra en experimentación con un grupo A experimental de estudiantes de 36 alumnos, los cuales se dividen a su vez en tres sub-grupos de 12 alumnos.

Existe un grupo B con 33 estudiantes que se considerará como grupo de control a los cuales se les impartirá clases de forma tradicional usando las mismas secuencias didácticas y se les proporcionarán los mismos problemas a resolver. Esto permitirá hacer comparativos que permitan detectar como la herramienta proporciona un mayor nivel de efectividad con respecto al aprendizaje de las matemáticas.

\section{Referencias.}

1. OCDE. http://www.oecd.org/centrodemexico/medios/Mexico\%20Country\%20Note SPANISH_final\%20GR1_EGcomments_02_12_2013\%20final.pdf. Bajado el 26/ mayo/ 2015 (2012)

2. Gonzalo Villareal, G.: Agentes inteligentes en educación. EDUTEC: Revista electrónica de tecnología educativa, Vol. 16, No. 4 (2003)

3. González González, C.S., Mora Carreño, A.: Técnicas de gamificación aplicadas en la docencia de Ingeniería Informática. Departamento de Ingeniería Informática, Universidad de La Laguna.

4. Barrón-Estrada, M.L., Zatarain-Cabada, R. Margarita Aranda-Ortega, H.E. Gómez-Pérez, José Mejía-Arredondo. Un tutor inteligente, afectivo y configurable para el aprendizaje de números naturales de 3er grado. Research in Computing Science, Vol. 77, pp. 45-54 (2014)

5. Clara Inés Peña, Jose-L Marzo, Josep Lluís de la Rosa, Ramón Fabregat: Un sistema de tutoría inteligente adaptativo considerando estilos de aprendizaje. Revista UIS ingenierías, 1(2) (2012)

6. Belavkin, Roman V.: The role of emotion in problem solving. Proceedings of the AISB'01 Symposium on emotion, cognition and affective computing, Heslington, York, England, pp. 49-57 (2001)

7. Aleven, V., McLaren, B. M., Sewall, J.: Scaling up programming by demonstration for intelligent tutoring systems development: An open-access web site for middle school mathematics learning. (2009)

8. González, C., Mora, A., Toledo, P.: Gamification in intelligent tutoring systems. Proceedings of the Second International Conference on Technological Ecosystems for Enhancing Multiculturality, pp. 221-225, ACM (2014)

9. Melis, E., Siekmann, J.: Activemath: An intelligent tutoring system for mathematics. Artificial Intelligence and Soft Computing-ICAISC 2004, pp. 91-101, Springer Berlin Heidelberg (2004) 
10. V. Aleven, B. M. McLaren, J. Sewall: Scaling up programming by demonstration for intelligent tutoring systems development: An open-access web site for middle school mathematics learning. IEEE Transactions on Learning Technologies, Vol. 2, Issue 2 (2009)

11. Arroyo, I.: Animalwatch: an arithmetic ITS for elementary and middle school students. Proceedings of the 5th International Conference on Intelligent Tutoring Systems, Montreal, Canada (2000)

12. D'Mello, S., Olney, A., Williams, C., Hays, P.: Gaze tutor: A gaze-reactive intelligent tutoring system. International Journal of human-computer studies, 70(5), 377-398 (2012)

13. D. M. Hart, I. Arroyo, J. E. Beck, B. P. Woolf, C. R. Beal: WhaleWatch : An intelligent multimedia math tutor. (1999)

14. Celso M. de Melo, Jonathan Gratch, Peter J. Carnevale: Humans versus Computers: Impact of emotion expressions on people's decision making. (2015) 